\title{
Multi-line TRL Calibration of Vector Network Analyzers: Classical versus Tensor Implementation
}

This paper was downloaded from TechRxiv (https://www.techrxiv.org).

\section{LICENSE}

CC BY 4.0

SUBMISSION DATE / POSTED DATE

$14-01-2022$ / 20-01-2022

CITATION

Buesa-Zubiria, Ana; Esteban, Jaime (2022): Multi-line TRL Calibration of Vector Network Analyzers: Classical versus Tensor Implementation. TechRxiv. Preprint. https://doi.org/10.36227/techrxiv.18319913.v1

$\mathrm{DOI}$

10.36227/techrxiv.18319913.v1 


\title{
Multi-line TRL Calibration of Vector Network Analyzers: Classical versus Tensor Implementation
}

\author{
Ana Buesa-Zubiria and Jaime Esteban
}

\begin{abstract}
A comprehensive comparison between two multi-line thru-reflect-line calibration techniques is provided. One of the methods is based on the classical combination of $N$ measurements following the Gauss-Markov theorem. The other one has been developed along this paper in a complete and detailed way using the recently published idea of considering each of the $N$ measurements as part of a tensor. The measurement of a calibration kit that covers from $500 \mathrm{MHz}$ to $20.5 \mathrm{GHz}$ has been carried out for both methods and the results discussed. From the analysis of the results, it seems that the tensor approach could show some advantages in the (not uncommon) cases where a strictly white and Gaussian noise is not the only perturbation of the measurements. On the other hand, the decomposition of the tensor requires a higher computational effort, which would better be reduced.
\end{abstract}

\section{INTRODUCTION}

In the field of microwaves, the characterization of the manufactured devices is of great interest for the correct operation of any system. Vector Network Analyzers (VNA) are essential for most high-frequency circuit characterizations, and their calibration is an essential step to obtain reliable measurements. Nowadays, the development of precise calibration techniques is of undeniable interest, as the specifications of radio frequency and microwave devices are increasingly demanding. Thus, many calibration techniques have been developed over the years $[2-6,8,9,11,14,16]$.

Let's focus on those calibrations that only consider the correction of linear errors throughout the measurements, so it is not necessary to know the source and nature of the non-linearity. Under this premise, one of the most common calibration techniques is the one known as thru-reflect-line (TRL) [3]. This is because the design of the calibration kit is simpler, as it uses a reflect instead of an open or a short circuit, which makes the kit less expensive to manufacture than other calibration techniques usually require. In case a higher degree of accuracy in the measurement results is needed, it is possible to increase the number of calibration standards in what is known as multi-line thru-reflect-line (MTRL) calibration. Recently, the basic ideas for the implementation of a MTRL calibration using a tensor decomposition have been published in $[12,13]$. However, in these papers the calibration procedure is not fully completed, and some results are obtained through generic non-linear optimization, instead of using the well-known optimal Gauss-Markov formulation presented in [2,8,9]. Moreover, the comparison made there with the classical formulation of the method is not fair, since in the application of the classical method the optimal combination of results is not used, neither in obtaining the propagation constant nor in the calculation of the calibration coefficients.

The aim of this work is to complete the description of the calibration process as based on the Canonical Polyadic Decomposition (CPD) algorithm [1], but using the optimal expressions presented in [2] instead of a generic non-linear optimization, and then compare the results with a careful implementation of the well-known method of [2]. The organization of this paper is as follows. First, Section 2 gives the main characteristics of the classical multi-line calibration method. Then, Section 3 completes and details the CPD multi-line calibration procedure. Section 4 provides a comparison

* Corresponding author: Ana Buesa-Zubiria (ana.buesa.zubiria@upm.es).

A. Buesa-Zubiria and J. Esteban are with the Information Processing and Telecommunications Center, Escuela Técnica Superior de Ingenieros de Telecomunicación, Universidad Politécnica de Madrid, 28040 Madrid, Spain. (e-mail: ana.buesa.zubiria@upm.es; jaime.esteban@upm.es). 
between the two implementations by measuring a microstrip MTRL kit covering the 0.5 to $20.5 \mathrm{GHz}$ frequency band. Finally, some conclusions are presented in Section 5.

\section{CLASSICAL MULTI-LINE CALIBRATION METHOD}

In the seminal MTRL article [9], an analysis of the error of TRL was derived, up to the first order and, based on the analysis of the measurement errors, the MTRL technique was established. Being [9] hard to follow, a subsequent publication that provides some understanding of the algorithm was presented in [2]. Unfortunately, typos in some equations make it difficult to apply them directly. Furthermore, the criteria for deriving the equations are not provided, assuming that the reader will make an intensive reading of the previous publications. As a consequence, it seems that a certain misunderstanding of this calibration method is widespread $[7,12,13]$ (some misconceptions will be commented on later). Therefore, the main objective of this section is to show the implementation options of the method, along with their implications in the final result, hoping to clarify any doubts that may arise about this calibration technique. The understanding of the classical calibration is mandatory in order to later understand its alternatives such as the use of CPD presented in Section 3.

\subsection{Determination of the Common Line}

In [9] a method is provided based on the combination of $N$ measurements following the Gauss-Markov theorem. For this, $N-1$ independent linear equations are obtained. Thus, one of the lines is necessarily used as a common line to form the different pairs that leads to the corresponding equations. The selection of the common line is based on minimizing the TRL error of the line pairs that can be formed, which implies that the sine of the difference in electrical length between the lines of the different pairs should be as large as possible. As a consequence, the common line must be redefined at each frequency.

To improve the robustness of the numerical calculation, the concept of effective phase difference is used for the determination of the common line:

$$
\Phi_{e f f}=\sin ^{-1}\left|\frac{e^{\gamma_{e s t} \Delta l_{i j}}-e^{-\gamma_{e s t} \Delta l_{i j}}}{2}\right|
$$

where $\gamma_{\text {est }}$ is a pre-estimation of the propagation constant of the lines that form the MTRL calibration kit, and $\Delta l_{i j}$ the difference in physical length between $i$-th and $j$-th lines.

Thus, for each frequency, the common line turns out to be the one that maximizes the lowest $\Phi_{\text {eff }}$ value of those obtained for all the lines with each possible common line. The determination of the common line is important as the theory and experiments show that it influences the accuracy of the calibration results. This influence will be shown at the end of this document by analyzing actual measurements.

\subsection{Determination of the Propagation Constant}

The evaluation of the propagation constant not only offers the possibility of shifting the calibration reference plane but is also necessary to complete the calibration, as it will be shown in Section 2.3. The estimation of the propagation constant will also be a useful tool for the evaluation of the calibration technique, so this section focuses on it.

In the procedure being described, $[2,9]$, it is necessary to calculate, at each frequency, the solution to the eigenvalue problems arising from the $N-1$ pair of lines that make use of the common line. In the same manner as in the conventional TRL [3], we have two measurements $i$ and $j$, for lines of lengths $l_{i}$ and $l_{j}$, respectively. Their wave-chain (or wave-cascading) matrices $\mathbf{M}^{i}$ and $\mathbf{M}^{j}$ can be written as:

$$
\begin{aligned}
& \mathbf{M}^{i}=\mathbf{X} \mathbf{T}^{i} \mathbf{Y} \\
& \mathbf{M}^{j}=\mathbf{X} \mathbf{T}^{j} \mathbf{Y}
\end{aligned}
$$

where $\mathbf{X}$ and $\mathbf{Y}$ represent the VNA non-idealities and $\mathbf{T}^{i}$ is the transfer matrix of the $i$-th line:

$$
\mathbf{T}^{i}=\left[\begin{array}{cc}
e^{-\gamma l_{i}} & 0 \\
0 & e^{+\gamma l_{i}}
\end{array}\right]
$$


So, we get that

$$
\mathbf{M}^{i j} \mathbf{X}=\mathbf{X} \mathbf{T}^{i j}
$$

with $\mathbf{M}^{i j}=\mathbf{M}^{j}\left(\mathbf{M}^{i}\right)^{-1}$ and $\mathbf{T}^{i j}=\mathbf{T}^{j}\left(\mathbf{T}^{i}\right)^{-1}$. Then, $\mathbf{M}^{i j}$ and $\mathbf{T}^{i j}$ are similar matrices, and therefore, they share their eigenvalues.

Once we have solved the eigenvalue problem for each pair of lines, its two solutions must be identified as $e^{-\gamma \Delta l_{i j}}$ and $e^{+\gamma \Delta l_{i j}}$, with $\Delta l_{i j}=l_{i}-l_{j}$. Although identification is an easy task in the absence of noise, in case of small disturbances, such as non-ideal connection repeatability, the small values of the attenuation constant often make identification a difficult task. So, to make the algorithm robust, a pre-estimation of the propagation constant of the lines, $\gamma_{e s t}$, is used. As the reader may realize, many identification algorithms can be developed but none of them influence the results of the calibration as long as the identification is done correctly. Once this identification is made, the $N-1$ pairs of eigenvalues allow to obtain $N-1$ propagation constants as

$$
\gamma_{i j}=\frac{\ln \left(e^{+\gamma \Delta l_{i j}}\right)-\ln \left(e^{-\gamma \Delta l_{i j}}\right)}{2 \Delta l_{i j}}
$$

Therefore, the algorithm obtains a single $\gamma_{i j}$ for each line pair, since both exponentials are combined to obtain a more precise result. Contrary to the belief of [13], the results extracted independently from the two exponentials cannot be exactly the same because the calculation of eigenvalues is carried out through the measurement, $\mathbf{M}^{i j}$, instead of the ideal wave-chain matrix $\mathbf{T}^{i j}$. It is true that in [9] it has been demonstrated that, under the measurement errors hypothesis, both propagation constants are identical up to the consideration of the first order error. But they may differ when more terms are considered.

Then we have to calculate, again for each of the frequencies, the estimation of the propagation constant, $\hat{\gamma}$, combining the $N-1$ propagation constants $\gamma_{i j}$ of (5). Their combination following the Gauss-Markov theorem is the main key of the multi-line algorithm. With it, it is possible to achieve the best unbiased linear estimation under a series of considerations about the nature of measurement uncertainties. This means, as it is said in [9], that the best estimation of the parameter must take into account all measurements (and not only the best line pair, as it seems to be read in [12]).

Let's define $\mathbf{L}$, size $(N-1) \times 1$, as the array containing all the $\Delta l_{i j}$ values and $\mathbf{G}$ as the size $(N-1) \times 1$ array with the $N-1$ values of $\gamma_{i j}$. If we name $\mathbf{V}$ of size $(N-1) \times(N-1)$ the measurement of the noise covariance we have that:

$$
\hat{\gamma}=\frac{\mathbf{L}^{H} \mathbf{V}^{-1} \mathbf{G}}{\mathbf{L}^{H} \mathbf{V}^{-1} \mathbf{L}}
$$

where the superindex $H$ stands for hermitian transpose.

The calculation of the $\mathbf{V}$ matrix is another key element that was presented in [9]. Realizing that the eigenvalue solution uses the difference between two measurements $\left(\mathbf{M}^{i j}\right)$, the noise covariance matrix must be filled in accordingly. Therefore, assuming that there is no correlation between the measurement of the different lines and that they are all equally noisy, we get:

$$
\mathbf{V}^{-1}=\frac{1}{\sigma_{k}^{2}}\left[\begin{array}{cc}
1-\frac{1}{N} & -\frac{1}{N^{\prime}} \\
-\frac{1}{N} & 1-\frac{1}{N}
\end{array}\right]
$$

where $\sigma_{k}$ is the variance of the noise in the individual line measurements, not being necessary its determination because of its appearance in the numerator as well as in the denominator of (6).

\subsection{Determination of the Correction Coefficients}

As in the first proposal of the TRL calibration technique, [3], the uncertainties of the non-ideal vector network analyzer are collected, as shown in (2), into two wave-chain matrices: $\mathbf{X}$ for port 1 and $\mathbf{Y}$ for port 2. The measurement of a device can be expressed as in (2), substituting the $\mathbf{T}^{i}$ by the device under test wave-chain matrix. The $\mathbf{X}$ and $\mathbf{Y}$ matrices can be written as:

$$
\mathbf{X}=R_{1}\left[\begin{array}{cc}
A_{1} & B_{1} \\
C_{1} & 1
\end{array}\right] \quad, \quad \overline{\mathbf{Y}}=R_{2}\left[\begin{array}{cc}
A_{2} & B_{2} \\
C_{2} & 1
\end{array}\right]
$$


where $\overline{\mathbf{Y}}$ is the reversed, left to right, $\mathbf{Y}$ matrix. Reversing a wave-chain matrix can be done by simply:

$$
\overline{\mathbf{Y}}=\left[\begin{array}{ll}
0 & 1 \\
1 & 0
\end{array}\right] \mathbf{Y}^{-1}\left[\begin{array}{ll}
0 & 1 \\
1 & 0
\end{array}\right]
$$

The main objective of reversing the matrix is to be able to use the same mathematical expressions for both ports.

The values of $B_{i}$ and $C_{i} / A_{i}$ with $i=\{1 ; 2\}$ in (8) are calculated for each of the $N-1$ pair of lines in a conventional way, making use of the solutions of the eigenvalue problems. Some care must be taken, since the obtained eigenvectors are not suitably ordered and scaled to conveniently represent the matrices $\mathbf{X}$ and $\mathbf{Y}$ of the VNA measurements (2). If required, the results of the eigenvalue sorting procedure described in Section 2.2 can be applied.

The final step is to combine the $N-1$ values of $B_{i}$ and $C_{i} / A_{i}$, accumulated in vectors $\mathbf{b}_{1,2}$ and $\left(\frac{\mathbf{c}_{1,2}}{\mathbf{a}_{1,2}}\right)$, at each frequency, through the expressions derived, again, from the best unbiased linear estimate:

$$
\begin{gathered}
\widehat{B}_{1,2}=\mathbf{h}^{T} \mathbf{V}_{B}^{-1} \mathbf{b}_{1,2} / \sigma_{B}^{2} \\
C_{1,2} / A_{1,2}=\mathbf{h}^{T} \mathbf{V}_{C}^{-1}\left(\frac{\mathbf{c}_{1,2}}{\mathbf{a}_{1,2}}\right) / \sigma_{C}^{2}
\end{gathered}
$$

where $\mathbf{h}$ is a vector whose elements all take the unit value and the superindex $T$ stands for transpose. Furthermore, $\sigma_{B}^{2}$ and $\sigma_{c}^{2}$, which are dividing into (10) instead of multiplying as it has been stated in [2], are the sum of all the elements in $\mathbf{V}_{B}^{-1}$ and $\mathbf{V}_{C}^{-1}$, respectively. Covariance matrices depend on the assumptions made about the nature of the noise measurement. In the classical MTRL these assumptions are:

- all lines are equally noisy,

- the noise is uncorrelated between different lines,

- uncertainties come exclusively from the differences between the connectors,

- connectors are symmetrically noisy.

With these assumptions we obtain the expressions of the $(m, n)$ terms of the covariance matrices, with $m, n \leq(N-1)$, similar to those of the original paper, but with the expressions written in a more compact form and where a typo has been corrected:

$$
\begin{aligned}
\left.V_{B}\right|_{m=n} & =\frac{\left|x_{m} / x_{c o m}\right|^{2}+\left|x_{c o m} / x_{m}\right|^{2}+2\left|x_{m} x_{c o m}\right|^{2}}{\left|x_{m} / x_{c o m}-x_{c o m} / x_{m}\right|^{2}} \\
\left.V_{B}\right|_{m<n} & =\frac{\left(x_{m} / x_{c o m}\right)\left(x_{n} / x_{c o m}\right)^{*}+\left|x_{c o m}\right|^{2} x_{m} x_{n}^{*}}{\left(x_{m} / x_{c o m}-x_{c o m} / x_{m}\right)\left(x_{n} / x_{c o m}-x_{c o m} / x_{n}\right)^{*}} \\
\left.V_{B}\right|_{m>n} & =\left.V_{B}\right|_{m<n} ^{*}
\end{aligned}
$$

and,

$$
\begin{aligned}
\left.V_{C}\right|_{m=n} & =\frac{\left|x_{m} / x_{c o m}\right|^{2}+\left|x_{c o m} / x_{m}\right|^{2}+\frac{2}{\left|x_{m} x_{c o m}\right|^{2}}}{\left|x_{m} / x_{c o m}-x_{c o m} / x_{m}\right|^{2}} \\
\left.V_{C}\right|_{m<n} & =\frac{\left(x_{c o m} / x_{m}\right)\left(x_{c o m} / x_{n}\right)^{*}+\frac{1}{\left|x_{c o m}\right|^{2} x_{m} x_{n}^{*}}}{\left(x_{m} / x_{c o m}-x_{c o m} / x_{m}\right)\left(x_{n} / x_{c o m}-x_{c o m} / x_{n}\right)^{*}} \\
\left.V_{C}\right|_{m>n} & =\left.V_{C}\right|_{m<n} ^{*}
\end{aligned}
$$

where $x_{k}=e^{-\hat{\gamma} l_{k}}, x_{c o m}=e^{-\hat{\gamma} l_{c o m}}$, and com is the index of the line chosen as common line.

As shown, the estimation of the propagation constant $\hat{\gamma}$ has been used to calculate the covariance matrices needed to combine the results of the $N$ measurements.

Once we get them, $A_{i}$ and $C_{i}$ must be derived as individual values, as well as $R$, which is the product of the $R_{i}$ factors in (8). For this purpose, the thru measurement together with the reflect measurements at both ports are used in a conventional way. It should be clarified that, in order to 
separate the factors $A_{i}$ and $C_{i}$, the thru measurement is considered as if its calibrated response were an ideal zero-length connection. Therefore, the measurement of the thru has more influence than the rest of the lines. However, this is not critical, since all differences between lines have already been optimally taken into account in the calculation of the quotient $\widehat{C_{i} / A_{i}}$ and the $\widehat{B_{i}}$ factor. Finally, to get the $R$ factor, the measurement of the thru is forced to be reciprocal.

\section{CPD IMPLEMENTATION}

In [13] the propagation constant of a line is obtained from the decomposition of a tensor which includes the data of $N$ simulations of $N$ lines that only differ in length. However, many of the details of the procedure are omitted or insufficient when it comes to real measurements instead of simulations. In addition, the calibration process is not fully described. Therefore, throughout this section, all the necessary details are given as well as the complete calibration method.

\subsection{Building the Tensor}

As an alternative to the method of Section 2, the measured data from all lines could be processed at the same time. Let us write $\mathbf{M}^{i}$ as

$$
\mathbf{M}^{i}=\underbrace{\left[\begin{array}{ll}
x_{11} & x_{12} \\
x_{21} & x_{22}
\end{array}\right] A\left[\begin{array}{ll}
p & 0 \\
0 & 1
\end{array}\right]}_{\mathbf{X}} \mathbf{P} \mathbf{T}^{i} \underbrace{\mathbf{P}}_{\mathbf{Y} B\left[\begin{array}{ll}
q & 0 \\
0 & 1
\end{array}\right]\left[\begin{array}{ll}
y_{11} & y_{12} \\
y_{21} & y_{22}
\end{array}\right]}
$$

where $\mathbf{P}$ is a permutation matrix and $A, p, B$ and $q$ are unknown scalar values. So,

$$
\begin{gathered}
\mathbf{M}^{i}=A B p q\left[\begin{array}{ll}
x_{11} y_{11} & x_{11} y_{12} \\
x_{21} y_{11} & x_{21} y_{12}
\end{array}\right] e^{\mp \gamma l_{i}}+ \\
A B\left[\begin{array}{ll}
x_{12} y_{21} & x_{12} y_{22} \\
x_{22} y_{21} & x_{22} y_{22}
\end{array}\right] e^{ \pm \gamma l_{i}}
\end{gathered}
$$

which can be written as:

$$
\begin{array}{r}
\mathbf{M}^{i}=\lambda_{1} e^{\mp \gamma l_{i}}\left[\begin{array}{l}
x_{11} \\
x_{21}
\end{array}\right]\left[\begin{array}{ll}
y_{11} & y_{12}
\end{array}\right]+ \\
\lambda_{2} e^{ \pm \gamma l_{i}}\left[\begin{array}{l}
x_{12} \\
x_{22}
\end{array}\right]\left[\begin{array}{ll}
y_{21} & y_{22}
\end{array}\right]
\end{array}
$$

or

$$
\mathbf{M}^{i}=\lambda_{1} e^{\mp \gamma l_{i}} \mathbf{a} \odot \mathbf{b}+\lambda_{2} e^{ \pm \gamma l_{i}} \mathbf{c} \odot \mathbf{d}
$$

where $\odot$ denotes the outer product between two column vectors $\mathbf{a} \odot \mathbf{b}=\mathbf{a b}^{T}$ being the superindex $T$ the transpose operation. Hence, the external product of two vectors results in a matrix. Extending this definition to tensors, each new vector outer product adds a new dimension to the result. Then, we can write:

$$
\mathcal{T}=\lambda_{1} \mathbf{a} \odot \mathbf{b} \odot \mathbf{e}_{1}+\lambda_{2} \mathbf{c} \odot \mathbf{d} \odot \mathbf{e}_{2}
$$

where $\mathbf{e}_{1}$ and $\mathbf{e}_{2}$ contain the propagation factors of the $N$ lines considered in the problem.

We can define the factor matrices:

$$
\begin{gathered}
\dot{\mathbf{X}}=\left[\begin{array}{ll}
\mathbf{a} & \mathbf{c}
\end{array}\right]=\left[\begin{array}{ll}
x_{11} & x_{12} \\
x_{21} & x_{22}
\end{array}\right]=\mathbf{X P} \frac{1}{A}\left[\begin{array}{cc}
1 / p & 0 \\
0 & 1
\end{array}\right] \\
\dot{\mathbf{Y}}^{T}=\left[\begin{array}{ll}
\mathbf{b} & \mathbf{d}
\end{array}\right]^{T}=\left[\begin{array}{ll}
y_{11} & y_{12} \\
y_{21} & y_{22}
\end{array}\right]=\frac{1}{B}\left[\begin{array}{cc}
1 / q & 0 \\
0 & 1
\end{array}\right] \mathbf{P Y}
\end{gathered}
$$


and

$$
\mathbf{D}=\left[\begin{array}{ll}
\mathbf{e}_{1} & \mathbf{e}_{2}
\end{array}\right]=\left[\begin{array}{cc}
e^{\mp \gamma l_{1}} & e^{ \pm \gamma l_{1}} \\
e^{\mp \gamma l_{2}} & e^{ \pm \gamma l_{2}} \\
e^{\mp \gamma l_{3}} & e^{ \pm \gamma l_{3}} \\
\vdots & \vdots \\
e^{\mp \gamma l_{N}} & e^{ \pm \gamma l_{N}}
\end{array}\right]
$$

The $\mathbf{D}$ matrix is written this way to show that in each column of $\mathbf{D}$ we can obtain either a positive or a negative exponential, as a result of the presence of the permutation matrix $\mathbf{P}$. But it is not possible that both types of exponentials are mixed in the same column, as it can be deduced from (16).

Then, with $\boldsymbol{\lambda}=\left[\begin{array}{ll}\lambda_{1} & \lambda_{2}\end{array}\right]$ :

$$
\mathcal{T}=[\boldsymbol{\lambda} ; \dot{\mathbf{X}}, \dot{\mathbf{Y}}, \mathbf{D}]
$$

which corresponds with the Canonical Polyadic Decomposition (CPD) operation. The CPD is a tensor decomposition that is unique, up to a permutation and scaling factors, when the sum of the factor matrices ranks, defined as the number of linearly independent columns, is greater or equal to $2 F+2$ being $F$ the number of summing elements in (16). So, in the MTRL calibration technique uniqueness is achieved. Therefore, if a tensor $\mathcal{T}$ containing the transmission parameters of $N$ lines of different lengths, but with identical launchers is formed, then the CPD gets the matrices $\dot{\mathbf{X}}, \dot{\mathbf{Y}}$ and $\mathbf{D}$ in equations (18) to (20). The matrices $\mathbf{X}$ and $\mathbf{Y}$, which define the VNA uncertainties, can be obtained once the permutation $\mathbf{P}$ and the scaling factors are found.

\subsection{CPD algorithm}

The CPD is usually implemented via a trilinear alternating least square algorithm, as it is the case for most of the procedures in [15]. Not because it is the best in convergence but because of its simplicity. The algorithm is based on minimizing the difference between each of the tensor dimensions with its value obtained by the product of its factor matrices. Two of the three factor matrices are fixed and the difference is calculated. Then another factor matrix is fixed. This procedure is repeated until one of the convergences criteria is achieved. In [17] it is shown that for the usual number of samples in signal processing applications the trilinear least square converges, or at least remains relatively close, to the Cramer Rao Bound in all the three dimensions for zero-mean identically distributed (i.i.d.) complex circularly symmetric Gaussian noise. Throughout this work, the trilinear alternating least square algorithm has been used, as well as others provided by [15], such as the non-linear least square or the algorithm based on the simultaneous generalized Schur decomposition. No differences in results have been detected among any of these algorithms.

\subsection{CPD Solution}

Note that the lines in (20) are sorted according to the order in which the measured data are included in $\mathcal{T}$, since permutation $\mathbf{P}$ does not affect it. However, the numerical result of (21) for $\mathbf{D}$ matrix can include two arbitrary scale factors: one of them multiplies the entire matrix, the other multiplies one column and divides the other. Because of the exponential form of the matrix terms, this second factor can be interpreted as a change of the reference plane. Therefore, some information about the line lengths should be used to fully determine the $\mathbf{D}$ matrix. In [13] an optimization is carried out, whose results are both the scale factors and the estimation of $\gamma$. In the implementation described here the obtainment of the propagation constant has been separated from the deduction of the scale factors in order to use the Gauss-Markov results. Thus, it can be ensured that the best linear estimation of the propagation constant can be found, at least when only considering the zero-mean additive white Gaussian noise (AWGN). Therefore, focusing throughout this section on the sole objective of determining the D matrix, a two-step normalization has been established. Firstly, just for simplicity and although it is not indispensable, the length of the line chosen as thru is set as zero. Then, each of the columns on (20) is normalized by the value on the position of the thru. This normalization gives special precedence to the accuracy of the result for the line chosen as thru. But the objective of the CPD is to make an 
approximation to the $\dot{\mathbf{X}}, \dot{\mathbf{Y}}$ and $\mathbf{D}$ values that best fit the tensor $\mathcal{T}$, without prioritizing any of the lines. This is why a second step has been included. The second step is based on an optimization, which finds the $\xi$ factor that best fits that all the elements in $\mathbf{e}_{\mathbf{1}}$, multiplied by their corresponding elements in $\mathbf{e}_{2}$, are equal to one. So, this second step can be seen as the fine-tuning of a factor $\xi$ that must divide both columns in (20). The optimization problem is stated as:

$$
\xi=\min _{\nu} \sum_{i=1}^{N}\left|\mathbf{e}_{i 1} \mathbf{e}_{i 2}-\nu^{2}\right|^{2}
$$

where the summation includes the thru, and the sign of $\xi$ is chosen so that the terms corresponding to the thru in the normalized $\mathbf{D}$ are close to +1 (and not to -1 ).

Furthermore, it is necessary to find the permutation that the CPD has introduced. As in the case of the classical MTRL, an estimation of the propagation constant has been used in our implementation. The main reason for such a decision is that, when considering low-loss transmission lines and noise, there is no trivial way to clearly distinguish which is the positive exponential and which is the negative one in the rows of $\mathbf{D}$. The permutation $\mathbf{P}$ is found by minimizing the distance between the exponential terms of $\mathbf{D}$ and the exponentials calculated with an estimation of the propagation constant. One must be cautious not to take a line that is too short or too long to find the permutation. A short one increases the possibility to misidentify the exponential factors. But, if the line is too long, identification errors also arise because of the indetermination introduced by the periodicity of the phase.

After all these steps, everything is ready to extract both the propagation constant and the correction coefficients of the multi-line TRL calibration technique.

\subsection{Propagation Constant by CPD}

In order to compare both methods in all their properties, the propagation constant has also been extracted with this formulation of the multi-line method. However, it should be noted that obtaining the propagation constant only allows shifting the calibration reference planes, i.e., it does not influence the calibration correction coefficients, as it can be derived from Section 3.5.

Once the permutation is found, the values of the propagation constant are calculated at each frequency, as given by each of the elements in both columns of $\mathbf{D}$ (excluding those corresponding to the thru). The possible phase jumps are then removed (the phase is unwrapped) so that the resulting propagation constant is continuous with frequency. As in the classical multi-line method, the propagation constants of the waves propagating in both directions are averaged to minimize the influence of second-order errors that may arise from differences between the connectors. In this way, $N-1$ values of $\gamma$ are obtained. Their combination can be optimally carried out using (6). This approach is better than the optimization proposed and used in [13], since at least (6) provides the best linear estimator.

\subsection{Correction Coefficients by CPD}

In order for the $\dot{\mathbf{X}}$ and $\dot{\mathbf{Y}}$ matrices to be consistent with a VNA measurement, the permutation found for $\mathbf{D}$ and the scaling factors mentioned in Section 3.3 are conveniently applied to these matrices. Then, the correction coefficients are found following the same steps as in Section 2.3, that is, making use of the thru measurement along with the measurements of the reflect at both ports of the VNA.

If the noise is considered to be zero-mean i.i.d. complex circularly symmetric Gaussian noise, the calibration coefficients in the $\mathbf{X}$ and $\mathbf{Y}$ matrices are essentially the same, whether the least-square alternate algorithm of the CPD has converged or the classical multi-line method has been followed, except for the scale factors introduced by the impossibility of independently determining the factors of the $R_{1} R_{2}$ product.

\section{COMPARISON}

A multi-line microstrip calibration kit (see Fig. 1), consisting of nine lines and an open end, has been manufactured using 30-mil thick ARLON25N as substrate. The line lengths have been chosen 


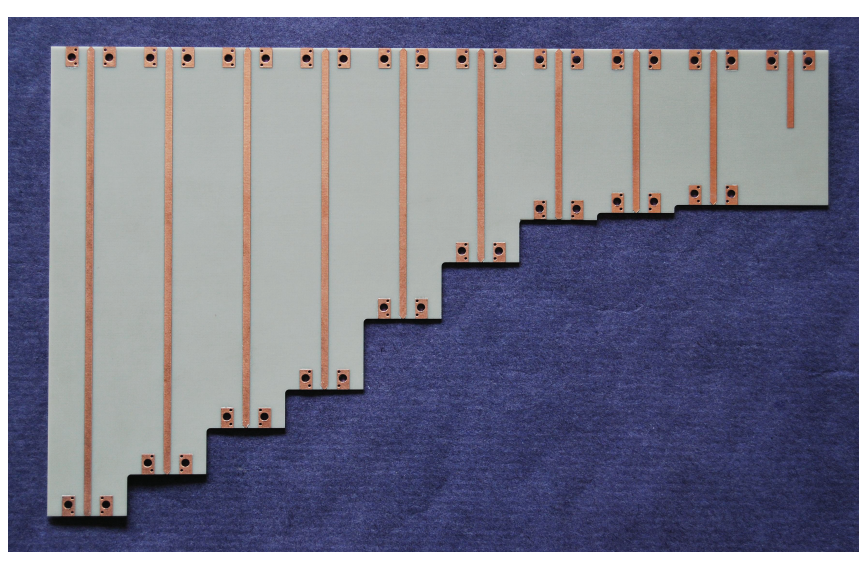

Figure 1. Manufactured calibration kit

to minimize the TRL calibration uncertainty in the range from $500 \mathrm{MHz}$ to $20.5 \mathrm{GHz}$, namely, 40, 42.1, 44, 54.9, 69.4, 87.5, 97.3, 109 and 119.5 millimeters. The microstrip open end used as reflect has been located $20 \mathrm{~mm}$ away from the launcher. Thus, the use of the $40 \mathrm{~mm}$ line as a zero-length thru places the reflect in the reference plane of the measurements.

Two comparisons are made throughout this section. Firstly, the circuit simulation of the calibration kit shows that both methods achieve the same results, as long as the only perturbation is a zero-mean i.i.d. complex circularly symmetric Gaussian noise. A check of the propagation constant estimation is also included. Secondly, the manufactured kit is measured, and the results using both the classical and the CPD methods are shown and discussed.

\subsection{Simulation of a nine-line MTRL calibration kit}

The frequency responses of the nine lines in the kit have been simulated with ADS using its circuital models for microstrip lines. Then, a complex Gaussian noise has been added to the resulting scattering parameters. Actually, both the real and imaginary parts of the noise follow a truncated Gaussian distribution, since the numerical values of the added uncertainties have been kept below $2 \sigma$ in the normal distribution. The choice of a circuital instead of an electromagnetic simulator avoids the accuracy errors introduced by the discrete mesh of full-wave simulators that cannot be represented by a linear error, even less by an AWGN.

To contrast the results of the two implementations (classical and CPD), the calibrated measurements obtained by both methods for one of the lines (for instance the one chosen as thru) have been compared by using a formulation similar to that given in [10, (8)]. It must be noted that in [10] a worst-case measurement error bounds are derived with respect to a benchmark calibration of known accuracy. Here, it is preferred to focus on the formulation of this paper, since no approximations (such as linearization of uncertainties) have been made.

For this, the matrix $\boldsymbol{\Delta}$ is defined as:

$$
\boldsymbol{\Delta}=20 \log \left|\mathbf{X}_{\text {classic }}^{-1} \mathbf{M}^{t} \mathbf{Y}_{\text {classic }}^{-1}-\mathbf{X}_{C P D}^{-1} \mathbf{M}^{t} \mathbf{Y}_{C P D}^{-1}\right|
$$

where $\mathbf{X}_{\text {classic }}$ and $\mathbf{Y}_{\text {classic }}$ are the matrices with the correction coefficients of the MTRL calibration using the classical implementation, the matrices with the subscripts $C P D$ are those of the CPD implementation, and $\mathbf{M}^{t}$ is the measured wave-chain matrix of the thru. If both calibrations were identical then $\boldsymbol{\Delta}=0$.

To check the proper coding of CPD procedure the elements of matrix $\Delta$ are shown in Fig. 2 in the case of no added noise. It is apparent that both methods obtain the same calibration coefficients, except for some numerical noise. In fact, the values of the elements $\boldsymbol{\Delta}_{11}$ and $\boldsymbol{\Delta}_{22}$ correspond to the minimum possible error $(-320)$, taking into account that double precision has been used in the calculations (around 16 significant decimal digits). 


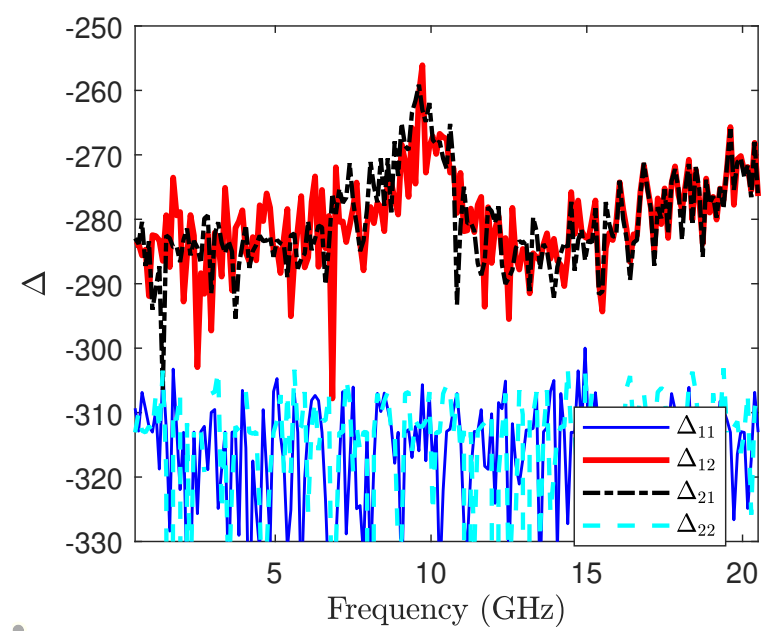

Figure 2. Comparison between the correction coefficients of the classical MTRL and the tensor-based method for noiseless simulations.
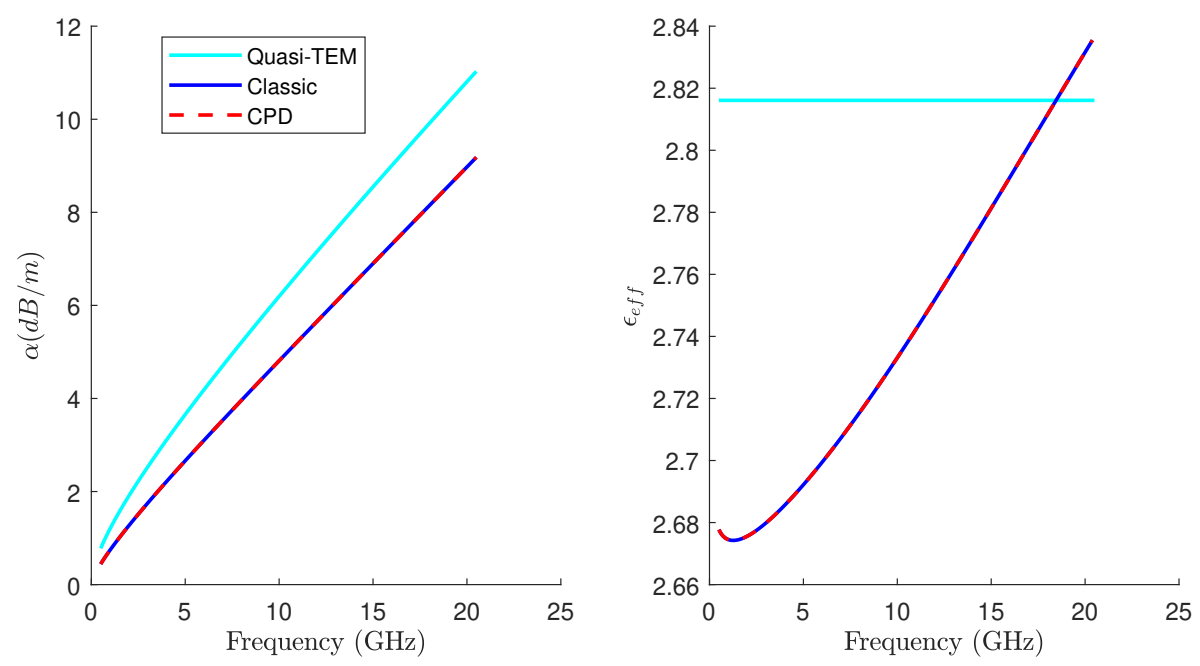

Figure 3. Attenuation constant and effective relative permittivity obtained by the classical MTRL and the tensor-based method for noiseless simulations.

It is also worth comparing the result of both formulations for the propagation constant. Fig. 3 shows the attenuation constant and the effective relative permittivity. Also shown in Fig. 3 is the preestimated propagation constant that is used in the identification step of the algorithms (see Section 2.2) and that has been borrowed from a quasi-static model of the microstrip. The coincidence of both formulations is confirmed, as well as the irrelevance of the pre-estimation of the propagation constant in the final results.

A zero-mean i.i.d. complex circularly symmetric Gaussian noise with standard deviation $\sigma=0.0283$ has then been added to the simulated $S$-parameters. The coefficients of the difference matrix (23) are shown in Fig. 4. The differences are higher than in the noiseless case, but remain at low values. Note that the CPD algorithm has some numerical noise associated with the convergence of the decomposition, while by definition the classical method is optimal when there are no error correlations.

The differences between the results for the attenuation constant and the effective permittivity, Fig. 5, are negligible. 


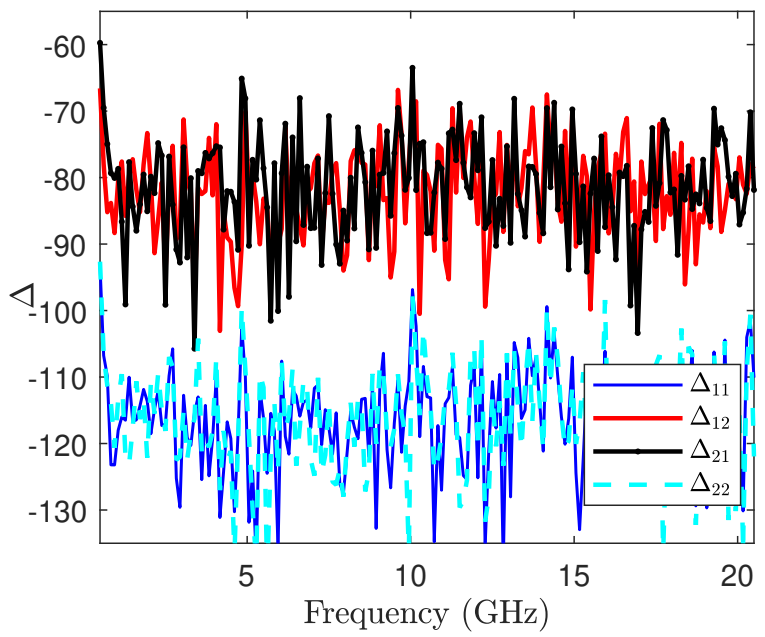

Figure 4. Comparison between the correction coefficients of the classical MTRL and the tensor-based method with AWGN of zero-mean and standard deviation $\sigma=0.0283$
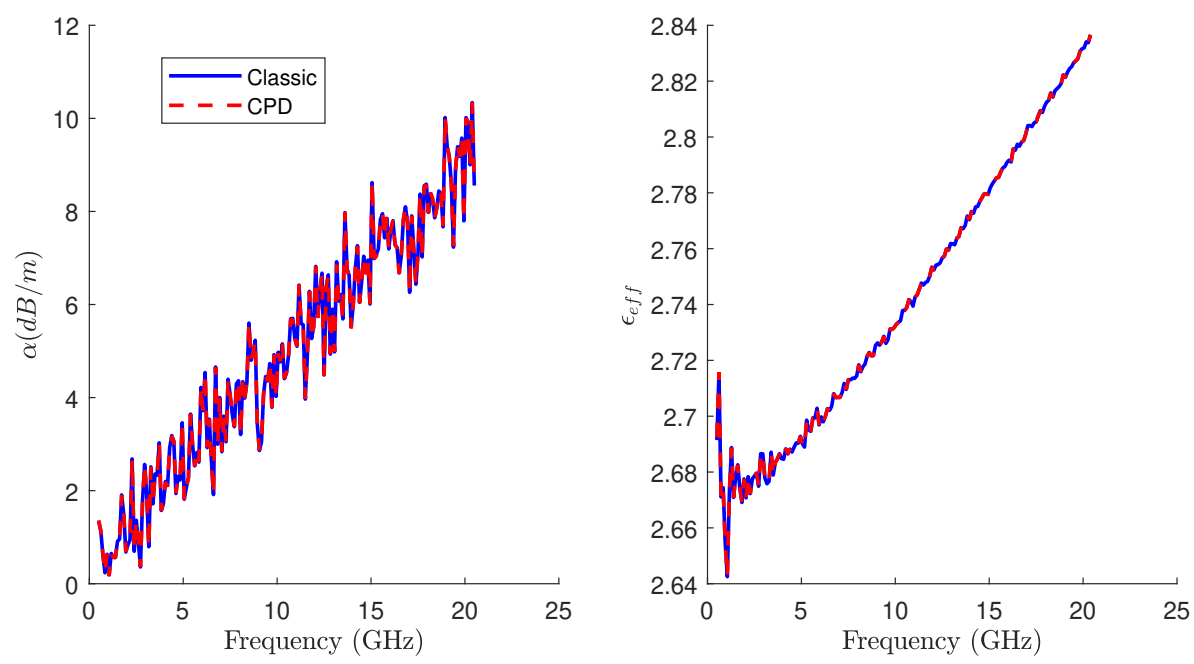

Figure 5. Attenuation constant and effective relative permittivity obtained by the classical MTRL and the tensor-based method with AWGN of zero-mean and standard deviation $\sigma=0.0283$

\subsection{Measurement of a Calibration Kit}

The multi-line calibration kit in Fig. 1, which includes the Southwest Microwave 292-04A-5 SMA female end-launch connectors on each side of the lines, has been measured with an Agilent N5230A VNA, and the resulting $S$-parameters have been processed with Matlab.

Results are shown throughout this section using the classical and CPD calibration formulations. Then, an analysis of the calibration results using different number of lines has been carried out to show their influence on the calibration coefficients.

It is worth insisting that both calibration methods achieve an optimum when considering zeromean AWGN. However, in the case of measurements, the noise is no longer of this nature and there are correlations between measurements. Therefore, it is not possible to determine which procedure offers the most accurate calibration.

For these measurements the differences in the calibration coefficients resulting from the two 


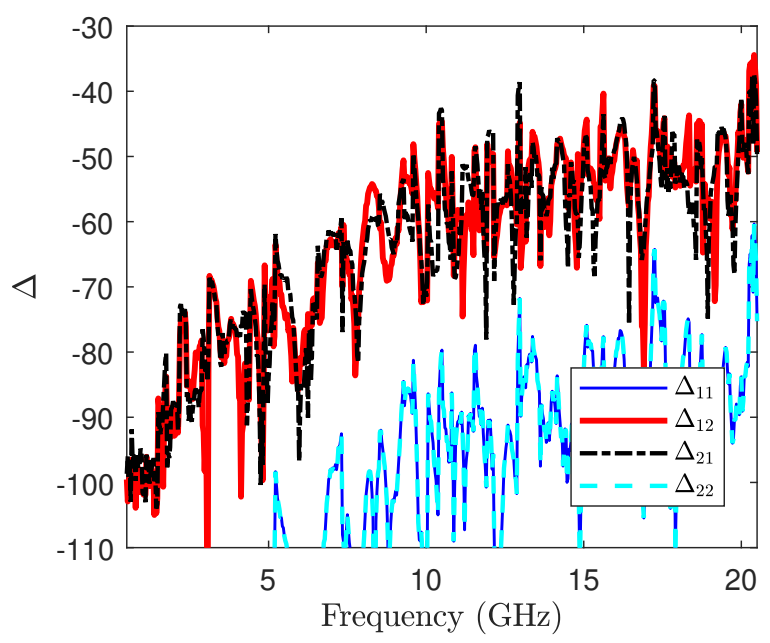

Figure 6. Comparison between the correction coefficients of the classical MTRL and the tensor-based method for the measured calibration kit.
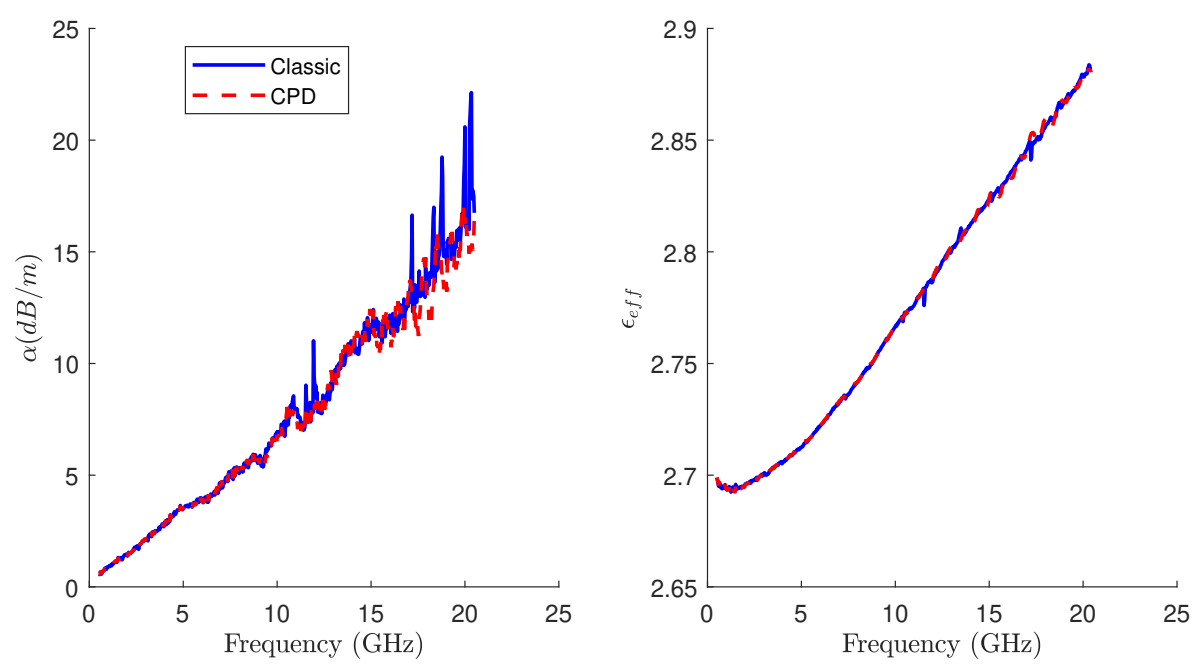

Figure 7. Attenuation constant and effective relative permittivity obtained by the classical MTRL and the tensor-based method for the measured calibration kit.

formulations are shown in Fig. 6, using the matrix of differences defined in (23). This figure shows that the results are quite similar to each other, except for the higher frequencies, at which the $\Delta_{12}$ and $\Delta_{21}$ terms reach values up to -35 . That value $\left(\Delta_{m n}=-35\right)$ implies differences in the second or third significant digit.

The propagation constant, as extracted from the measurements, is shown in Fig. 7. Its real part, the attenuation constant, is the most sensitive parameter. The results for the attenuation constant using the classical formulation show some spikes (see Fig. 7), more numerous and of greater amplitude at the higher frequencies, which do not appear with the CPD approach. The classical formulation also shows some spikes in the effective permittivity (Fig. 7), although of much lesser amplitude than in the case of the attenuation constant, that do not appear with the tensor-based method (only a ripple that increases in amplitude with frequency is appreciable).

Let's first focus on determining the nature of the peaks that occur when using the classical implementation of MTRL. For this purpose, the results of the attenuation constant have been calculated for three different common lines. The first common line is the optimal, as given by the maximization of the lowest $\Phi_{e f f}$ in (1); the second line is the second-best in the maximization of $\Phi_{e f f}$, and so on. 


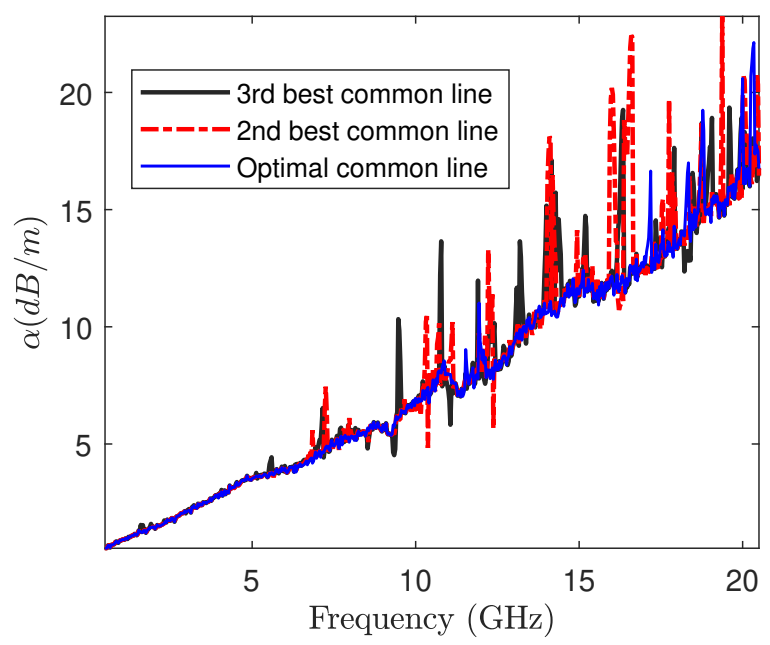

Figure 8. Attenuation constant obtained by the classical MTRL calculated with different common lines for the measured calibration kit.
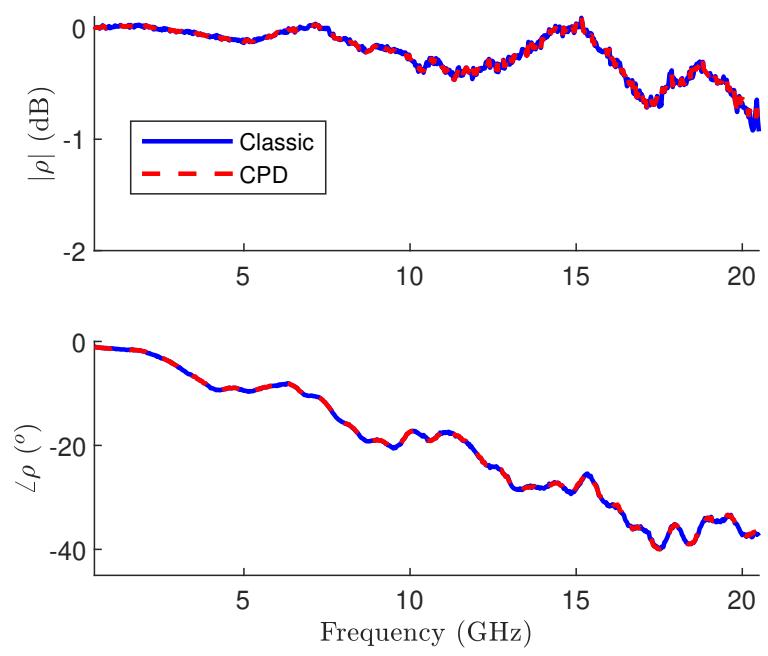

Figure 9. Calibrated reflection coefficient of the reflect using both the classical MTRL and the tensorbased method.

Fig. 8 shows that the sensitivity of the measurements to the chosen common line is very high when actual measurements are analyzed, which is not the case when dealing with simulations. Although with some caution, since both formulations of the MTRL calibration have been geared to the only presence of AWGN, it could be stated that the CPD has the advantage, when dealing with real measurements, that there is no need for a common line in the determination of $\mathbf{D}$ in (20). It should be noted that the propagation constant is in both implementations the best linear estimator from the set of eigenvalues obtained. However, this is not the case when obtaining the eigenvalues from which the best estimation of the propagation constant is extracted. It should be mentioned that both calibration procedures show an increase in ripple as the measurement frequency increases. The authors relate this phenomenon to the response of the connectors.

To complete the classical vs. CPD comparison, Fig. 9 shows the measurement of the reflection coefficient of the reflect, calibrated with the results of the two procedures. The results for this measurement are very similar.

An interesting issue may be to analyze the effect of adding more and more lines to a MTRL calibration kit. The aim of this analysis is to see its influence on the ripple that appears at high 


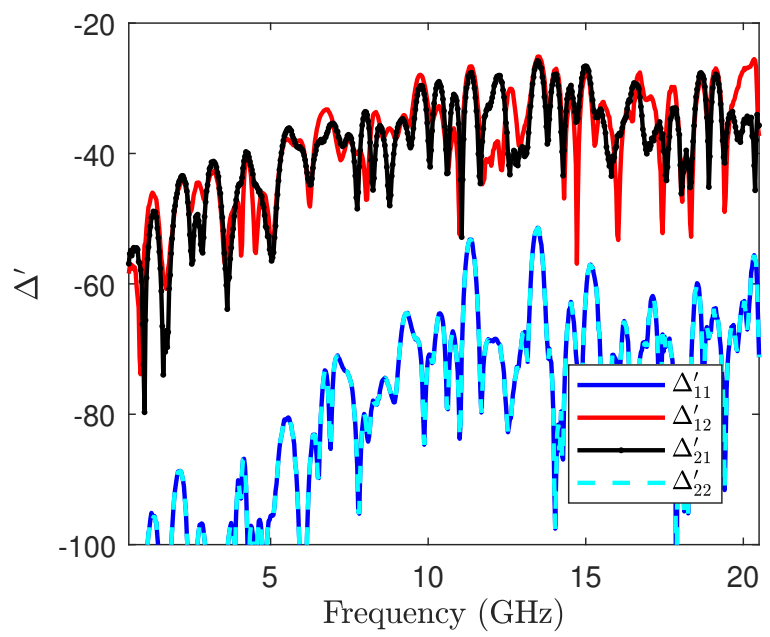

Figure 10. Comparison between the correction coefficients of the tensor-based MTRL approach, with four lines and with nine lines, using the measured calibration kit.

frequencies for the more sensitive parameter, the attenuation constant. It has already been shown that the classical method is very sensitive to the choice of the common line, so it will also be sensitive to a change in the number of lines used during calibration. Therefore, only the tensor-based method has been explored for this purpose. Four subsets of lines have been formed from the kit in Fig. 1.

The lines involved in each calibration subset have been chosen to keep the TRL first term error as constant as possible in the calibration bandwidth (from $500 \mathrm{MHz}$ to $20.5 \mathrm{GHz}$ ) and to keep at least one of the electrical lengths of the lines, $\theta$, between the usual limits $60^{\circ}<\theta<120^{\circ}$. Obviously, it is expected that when fewer lines are used in the calibration the error will increase in some frequency ranges. The subsets of calibration lines that have been used along this analysis are:

- The full set of nine lines.

- The six lines of lengths 40, 44, 54.9, 69.4, 87.5 and $109 \mathrm{~mm}$.

- The five lines of lengths 40,44, 54.9, 69.4 and $119.5 \mathrm{~mm}$.

- The four lines of lengths 40, 44, 87.5 and $109 \mathrm{~mm}$.

Using the measurements already made, the CPD approach to MTRL calibration has been applied to each subset, with the results discussed below. To simplify the comparison and the calculation of the MTRL coefficients the shortest line $(40 \mathrm{~mm})$ has been used in all cases as thru (which is assigned zero length) to maintain the phase reference plane at the location of the reflect.

In Fig.10 the elements of matrix $\boldsymbol{\Delta}^{\prime}$ are shown. Similar to (23), this matrix is the difference between the calibrated measurement of the thru using the CPD implementation, but when different number of lines have been used (including the one chosen as thru). It is defined as

$$
\boldsymbol{\Delta}^{\prime}=20 \log \left|\mathbf{X}_{9}^{-1} \mathbf{M}^{t} \mathbf{Y}_{9}^{-1}-\mathbf{X}_{4}^{-1} \mathbf{M}^{t} \mathbf{Y}_{4}^{-1}\right|
$$

where $\mathbf{X}_{9}$ and $\mathbf{Y}_{9}$ are the matrices with the correction coefficients of the MTRL calibration using the whole nine-lines set, while $\mathbf{X}_{4}$ and $\mathbf{Y}_{4}$ are the same matrices when the subset of four lines is used.

The differences of using four or nine lines are greater than those given by the choice of the calibration method as the reader can verify through the comparison of Figs. 6 and 10. Therefore, it can be concluded that the choice of the algorithm, classical or CPD, is of less relevance when only the results of the calibration are considered.

Focusing again on the most sensitive parameter, Fig. 11 shows the attenuation constant obtained with subsets of different number of lines. It should be highlighted that the more lines used, the less variability there is in the measurement. Figs. 11 and 12 compare the results for the effective relative permittivity and for the calibrate reflection coefficient of the reflect, respectively. In this particular case there seems to be virtually no difference in calibration accuracy when six or more lines are used. 

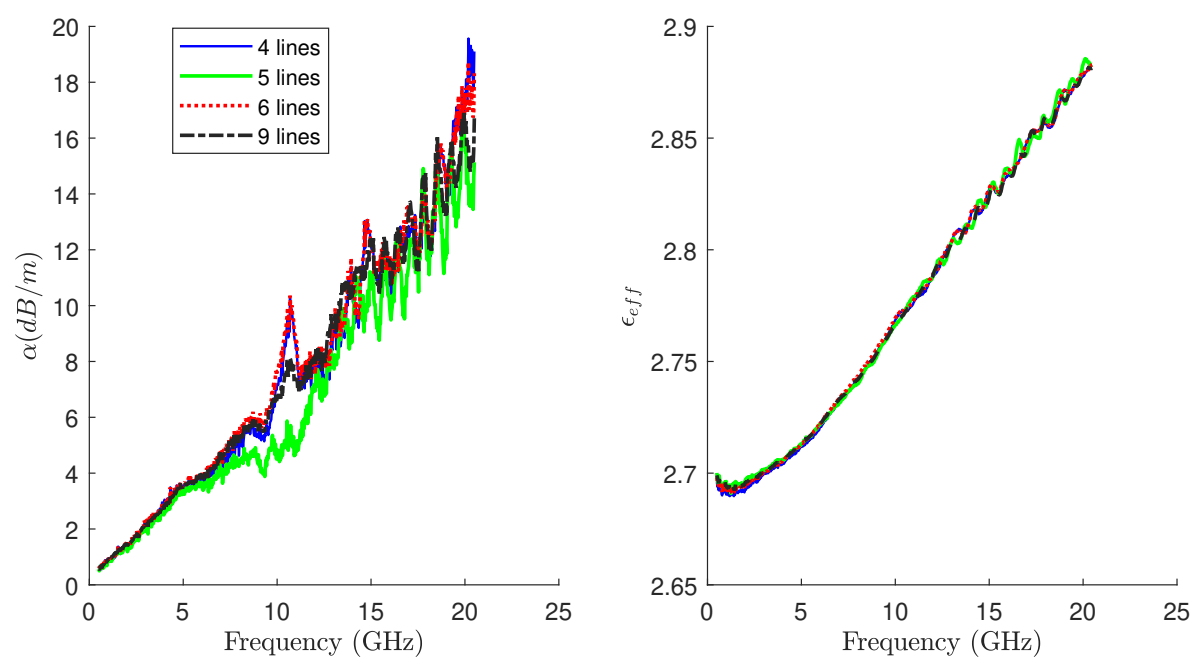

Figure 11. Attenuation constant and effective relative permittivity obtained by the tensor-based MTRL approach, with different number of lines, using the measured calibration kit.
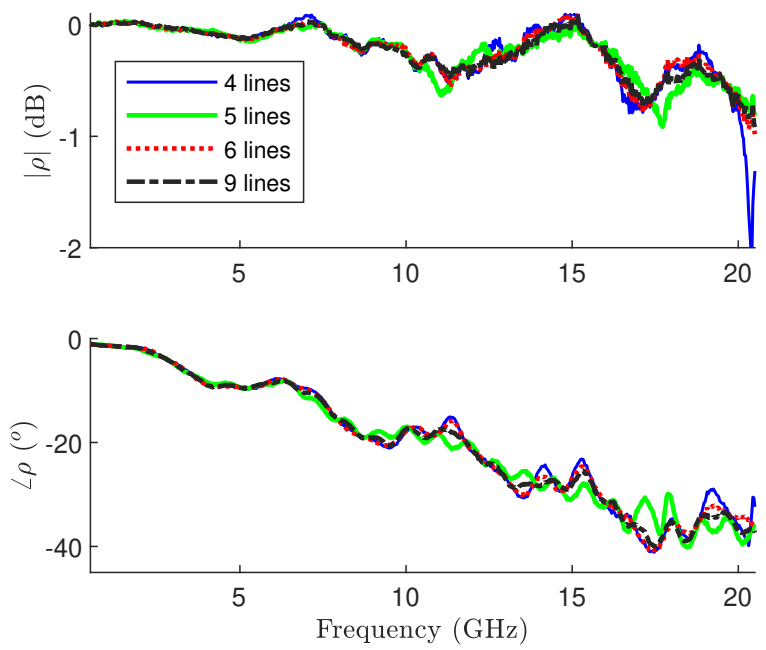

Figure 12. Calibrated reflection coefficient of the reflect obtained by the tensor-based MTRL approach, with different number of lines, using the measured calibration kit.

\section{CONCLUSIONS}

Through the rigorous completion of the MTRL calibration technique by tensor decomposition, it has been confirmed that both the classical and the tensor-based MTRL algorithm are essentially identical when considering a zero-mean i.i.d. complex circularly symmetric Gaussian noise as the only perturbation. When considering other types of noise and correlations (as in measurements) then the algorithms diverge, not being possible to decide which of them is more accurate, since there is no optimal estimator to check with. However, there are some spikes in the resolution of the eigenvalue problem by means of the classical implementation, which the CPD avoids. Nonetheless, it has been shown that the differences between the calibration coefficients of the two methods are negligible compared to the differences that arise from the selection of the number of lines involved in the calibration.

Additionally, a major difference in their implementations must be highlighted. The classical method, relying on an eigenvalue decomposition, always has an exact solution, regardless of the nature of the error affecting the standards, which is not the case with CPD. The CPD must find the error 
matrices that fit the measurements of all lines simultaneously. This process can be tedious for a large number of lines, and there is no exact solution when the noise is considered. The computation time required by the CPD should certainly be reduced by using a more complex and efficient algorithm than those used throughout this study (mainly the trilinear alternating least square algorithm). This would make the tensor-based calibration method an attractive alternative for the implementation of multi-line TRL calibration.

\section{ACKNOWLEDGMENT}

This work was supported by the Spanish Government under Grant PID2020-116968RB-C33 (DEWICOM) funded by MCIN/AEI/10.13039/501100011033 (Agencia Estatal de Investigación) and by the European Space Agency under contract 4000135255/21/NL/GLC/my.

\section{REFERENCES}

1. P. Comon. Tensors: A brief introduction. IEEE Signal Process. Mag., 31(3):44-53, May. 2014.

2. D. C. DeGroot, J. A. Jargon, and R. B. Marks. Multiline TRL revealed. In 60th ARFTG Conf. Dig., pages 131-155, Dec. 2002.

3. G. F. Engen and C. A. Hoer. Thru-reflect-line: An improved technique for calibrating the dual six-port automatic network analyzer. IEEE Trans. Microw. Theory Techn., 27(12):987-993, Dec. 1979.

4. H-J. Eul and B. Schiek. A generalized theory and new calibration procedures for network analyzer self-calibration. IEEE Trans. Microw. Theory Techn., 39(4):724-731, Apr. 1991.

5. A. Ferrero and U. Pisani. Two-port network analyzer calibration using an unknown 'thru'. IEEE Microw. Guided Wave Lett., 2(12):505-507, Dec. 1992.

6. C. A. Hoer and G. F. Engen. On-line accuracy assessment for the dual six-port ANA: Extension to nonmating connectors. IEEE Trans. Instrum. Meas., IM-36(2):524-529, Jun. 1987.

7. B. Hofmann and S. Kolb. A multistandard method of network analyzer selfcalibration-generalization of multiline TRL. IEEE Trans. Microw. Theory Techn., 66(1):245-254, Jan. 2018.

8. R. B. Marks. Multi-line calibration for MMIC measurement. In 36th ARFTG Conf. Dig., volume 18, pages 47-56, Nov. 1990.

9. R. B. Marks. A multiline method of network analyzer calibration. IEEE Trans. Microw. Theory Techn., 39(7):1205-1215, Jul. 1991.

10. Roger B. Marks, Jeffrey A. Jargon, and John R. Juroshek. Calibration comparison method for vector network analyzers. In 48th ARFTG Conference Digest, volume 30, pages 38-45, 1996.

11. S. Rehnmark. On the calibration process of automatic network analyzer systems (short papers). IEEE Trans. Microw. Theory Techn., 22(4):457-458, Apr. 1974.

12. Y. Rolain, M. Ishteva, E. Van Nechel, and F. Ferranti. Multi-line TRL revisited. In 85th Microw. Meas. Conf. (ARFTG), pages 1-3, May. 2015.

13. Y. Rolain, M. Ishteva, E. Van Nechel, and F. Ferranti. A tensor-based extension for the multi-line TRL calibration. IEEE Trans. Microw. Theory Techn., 64(7):2121-2128, Jul. 2016.

14. H. V. Shurmer. Calibration procedure for computer-corrected S parameter characterisation of devices mounted in microstrip. Electron. Lett., 9(14):323-324, Jul. 1973.

15. N. Vervliet, O. Debals, L. Sorber, M. Van Barel, and L. De Lathauwer. Tensorlab 3.0, Mar. 2016.

16. D. F. Williams and R. B. Marks. LRM probe-tip calibrations using nonideal standards. IEEE Trans. Microw. Theory Techn., 43(2):466-469, Feb. 1995.

17. Xiangqian Liu and N. D. Sidiropoulos. Cramer-rao lower bounds for low-rank decomposition of multidimensional arrays. IEEE Trans. Signal Process., 49(9):2074-2086, Sep. 2001. 